\title{
Failure modes analysis of railway wheel
}

\author{
Marius-Adrian Spiroiu ${ }^{1,{ }^{*}}$ and Mircea Nicolescu $^{2}$ \\ ${ }^{1}$ University Politehnica of Bucharest, Department of Rolling Stock, Splaiul Independenţei 313, \\ Bucharest, Romania \\ ${ }^{2}$ Romanian Railway Investigating Agency, Calea Griviţei 393, Bucharest, Romania
}

\begin{abstract}
In this paper are analysed the wheel failure modes, based on real field data, obtained during a period of a year of operation of freight wagons. The failure modes and their occurrence absolute frequencies are identified and a Pareto analysis is carried out. In the case of the four major failure modes a total time on test transform is performed and the failure probabilistic models are estimated, based on the Weibull distribution. The failure rates of the four failure modes are close to be constant, thus the behaviour of the railway wheel - in its normal life - is close to that of a non-ageing system. This conclusion is in line with the general theory regarding the behaviour of systems during their normal/useful life.
\end{abstract}

\section{Introduction}

A railway wheelset performs several essential functions by supporting the vehicle weight, by transmitting its traction and braking forces to the rails and by providing the vehicle guidance on the track. Under these circumstances, it is obvious that the wheelset reliability is a fundamental issue. A wheelset failure during operation may have important social and/or economic implications and - in the worst-case scenario - it may lead to important material damage and even to fatalities [1,2]. Also, wheel failure may have a negative impact on other elements of the vehicle (e.g. suspension) and of the track (e.g. rail).

Given its importance, many studies are approaching the wheel or wheelset reliability topic. For example, in [3] is presented a study on the methodology of fatigue reliability degradation of railroad wheels, considering fatigue crack initiation and propagation. The fatigue crack issue is addressed also in [4], based on a finite element analysis of wheel-rail contact and in [5], where are studied the cracks found on the wheel surface of an underground train. In [6] is investigated the influence of wheel material structure and properties on the wheel rim cracking caused by the fatigue process, while in [7] is performed an experimental study regarding the damages assessment in wheel-rail contact.

Some of the papers on this topic are focusing on the relation between reliability and the preventive maintenance strategy. For example, in [8] is presented a study regarding the use of degradation data in customizing the preventive maintenance strategy for locomotive wheelsets.

It should be noted that there are very rare the scientific papers presenting systematically the wheels failure modes or in which is derived a reliability probabilistic model of the

\footnotetext{
*Corresponding author: marius_spiroiu@yahoo.com
} 
railway wheel. Under these circumstances, the present paper aim is to analyse the failure modes of railway wagon wheels and to estimate their reliability probabilistic models, the analysis being made exclusively based on real field data.

\section{Wheel failure modes statistic}

The failure modes analysis in this paper is based on real field empirical data, observed in actual operations of freight wagons. The data were collected in the period of useful (normal) life of the units (the stable failure period). The sample consisted of 1802 units and, during the observation period of one year, there were found 137 cases of failure.

Before presenting the statistics of the failure modes identified during the observation period, there are necessary some additional details about the overall structure and the particularities of the railway wheels. The aim of this is to better understand the nature of each observed failure mode. The considered freight wagons wheels are tyred wheels, i.e. fitted with a steel hoop (tyre) on the outside of the wheel centre. The tyre has a rolling surface (tread) - the wheel-rail contact area -, and a flange on the inner side of the wheel, as a safety element [2]. In terms of proper operation, one specific feature of the railway wheels is that their peripheric geometric shape is very important for providing vehicle guidance, thus ensuring the traffic safety from the point of view of avoiding derailment. For this reason, the observed wheel failure modes are not only of the classical type for mechanical systems (cracks, fractures, deformations, etc.) but also ones related to the geometrical shape of the wheel profile (the cross-sectional profile of the wheel periphery). The latter consists of non-compliance of the prescribed (mandatory) limits of some essential dimensions of the wheel rolling profile, most of them referring to the flange, which is the key element in terms of guidance safety.

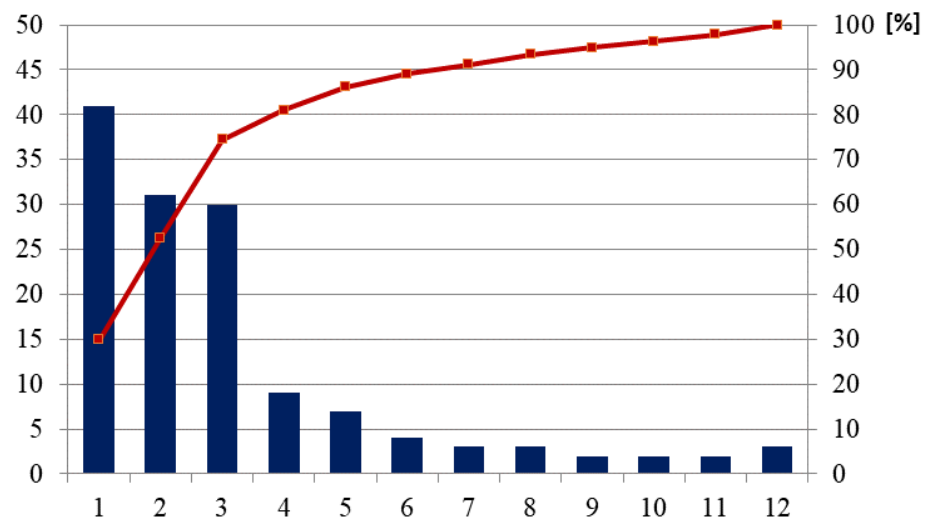

Fig. 1. Railway wheel failure modes: absolute frequency and cumulative percentage.

In Figure 1 are presented the recorded wheel failure modes, under the form of a Pareto chart. The failure modes are on the horizontal axis and the vertical axes are for the absolute frequencies of failure modes occurrence (left) and for cumulative percentage (right). The failure modes represented by the numbers on the horizontal axis are: 1 - wheel flat; 2 loosened tyre; 3 - scaled wheel (tread build-up); 4 - wheel diameter below the prescribed limit; 5 - thin flanges (flange thickness below the prescribed limit); 6 - high flanges (flange height over the prescribed limit); 7 - tread indentations; 8 - overheated wheels; 9 - steep flanges $\left(\mathrm{q}_{\mathrm{R}}\right.$ dimension, as a measure for flange angle, below the prescribed limit); 10 - tyre thickness below the prescribed limit; 11 - cracked tyre; 12 - other (3). The failure modes 
absolute frequencies are represented in the diagram by vertical columns, in descending order and the cumulative relative frequency is represented by the ascending broken line.

It can be seen that the Pareto principle applies to a good extent in the case of the analysed data: the first 4 (out of 14) most frequent failure modes are causing $81 \%$ of the total failures of the railway wheel; also, the first 3 modes (which is $21 \%$ out of 14 ) are producing $74.5 \%$ of the total failures.

In the present paper, it is considered that failure modes $4,5,6,9,10$ (using the stated above designation corresponding to Figure 1) may be included in a single category for subsequent analysis. They are all dimensional deviations from the prescribed limits and they all have the same cause: the tyre wear because of the friction between wheel and rail. Consequently, from here on, they will be referred to as a single failure mode: 'tyre wear'. Adding this new-defined failure mode to the first three most frequent failure modes (wheel flat, loosened tyre, scaled wheel), are obtained four failure modes which are accountable for a total of 126 (out of 137) failure cases.

\section{The total time on test transform analysis}

When a sample of identical units is tested or observed in operation, the units will fail at different times, so, at any moment of time during the test, some units will be still in operation and some units will be defective. The total time on test (TTT) statistic is defined as the sum of the operating times of all units, regardless of whether they are still in service (units without failure) or not (units that have failed) [9].

Considering a sample of $n$ units put to test at time 0 and given their respective failure times arranged in ascending order, $t_{1} \leq t_{2} \leq \ldots \leq t_{n}$, the empirical TTT up to the $i^{\text {th }}$ order statistic can be written as $[1,9]$ :

$$
\operatorname{TTT}(n, i)=\sum_{j=1}^{i} t_{j}+(n-r) t_{i},
$$

Which is the total time on test of the $n$ tested units, accumulated up to the $i^{\text {th }}$ failure time.

On the basis of the TTT can be obtained the so-called TTT transform plot, which has the property that the slope of the graphical representation in any point is the inverse of the failure rate at that point [9]. Consequently, the shape of the TTT transform plot provides information about the failure rate. The scaled TTT transform is defined by the ratio [9]:

$$
T_{s}(F)=\frac{T T T(n, i)}{T T T(n, n)},
$$

Where $F$ is the empirical failure probability. In the case of incomplete data, assuming that the test ends after the $k^{\text {th }}$ failure, $\operatorname{TTT}(n, k)$ is used instead of $\operatorname{TTT}(n, n)$ in equation (2), with $i=1 \ldots k$. The plot of $T_{s}$ versus $F$ is the scaled TTT transform curve, based on which can be identified the evolution in time of the failure rate: if the transform curve is concave, this indicates an increasing failure rate, i.e. a positive ageing (NBU - 'new better than used'); if the transform curve is convex, this indicates a decreasing failure rate, i.e. a negative ageing (NWU - 'new worse than used'); a straight line signifies a constant failure rate (no ageing situation).

Using equations (1) and (2), for the case of incomplete data, are obtained the total time on test transform for the four major wheel failure modes. The resulting plots are presented in Figure 2. Each point on the graph corresponds to the failure of a unit which occurred in the respective failure mode. It can be seen that, although there are peculiarities of each of 
the four graphical representations, they all have as a common feature a low curvature. This indicates that the failure rates associated with the failure modes have not a significant variation over time, so the behaviour of the railway wheel, from the point of view of the analysed failure modes, is close to that of a non-ageing system.
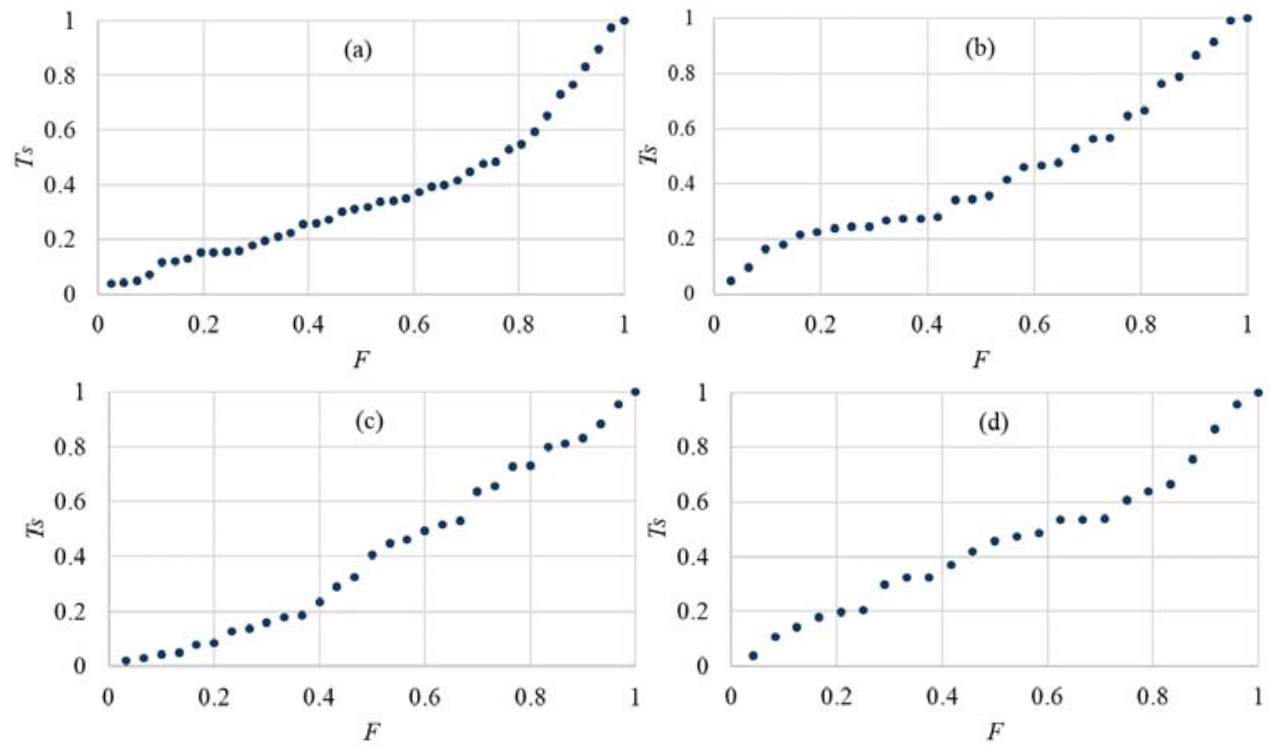

Fig. 2. Total time on test transform plot: wheel flat (a); loosened tyre (b); scaled wheel (c); tyre wear (d).

This finding was somehow to be expected, the explanation for it being that the data concerning the railway wheel failure were collected in the period of its useful life, during which the failure rate is stable. This hypothesis is to be confirmed by the analysis regarding the estimation of the failure mode probabilistic model.

\section{Failure modes probabilistic models}

In this section are estimated the probabilistic models for each of the four predominant wheel failure modes, using the Weibull distribution. The equation of reliability $R(t)$ in the case of the two-parameter Weibull distribution is given by [1]:

$$
R(t)=\exp \left[-\left(\frac{t}{\eta}\right)^{\beta}\right],
$$

Where $t$ is the time, $\beta$ and $\eta$ are the shape and the scale parameters, respectively.

In the theory of reliability there are several methods for estimating the parameters of failure mathematical models, of which the most commonly used are the maximum likelihood method, the method of moments and the regression method. In the present paper it is applied the latter one, which is based on the linearization of the Weibull probability law. By applying twice the natural logarithm in both members of equation (3) it is obtained:

$$
\ln \ln \frac{1}{R(t)}=\beta \ln t-\beta \ln \eta
$$


Denoting

$$
Y=\ln \ln \frac{1}{R(t)} ; X=\ln t
$$

The Weibull probability law can be expressed in a linear form:

$$
Y=a_{0}+a_{1} X
$$

Therefore the linear regression can be used to estimate the parameters of Weibull distribution. The regression coefficients $a_{1}$ and $a_{0}$ are derived using the Least Squares Method and the estimations of parameters $\beta$ and $\eta$ are given by:

$$
\hat{\beta}=a_{1} ; \hat{\eta}=\exp \left(-\frac{a_{0}}{a_{1}}\right) \text {. }
$$

Applying the method mentioned above and using the experimental data related to the four major railway wheel failure modes, the probabilistic mathematical models are derived. The parameters defining the Weibull model for each failure mode are presented in Table 1.

Table 1. Estimated Weibull distribution parameters.

\begin{tabular}{|ccc|}
\hline Failure mode & $\beta$ & $\eta$ [days] \\
\hline wheel flat & 1.019 & 7669 \\
loosened tyre & 1.179 & 8551 \\
scaled wheel & 0.753 & 73038 \\
tyre wear & 1.067 & 16973 \\
\hline
\end{tabular}

Of the two parameters, the most significant is the shape parameter $\beta$, which has a decisive influence on the failure rate. If $\beta=1$, a constant failure rate is obtained and, in this case, the exponential distribution can be used to model the reliability. It can be seen in Table 1 that the $\beta$ values are generally close to 1 , so the failure rates of the four failure modes have not important variations over time (i.e. are almost constant). These results confirm the previous analysis on the total time on test transform, as well as the general theory regarding the behavior of systems during their normal/useful life. The four wheel failure probabilistic models of the form given by equation (3) are plotted in Figure 3.

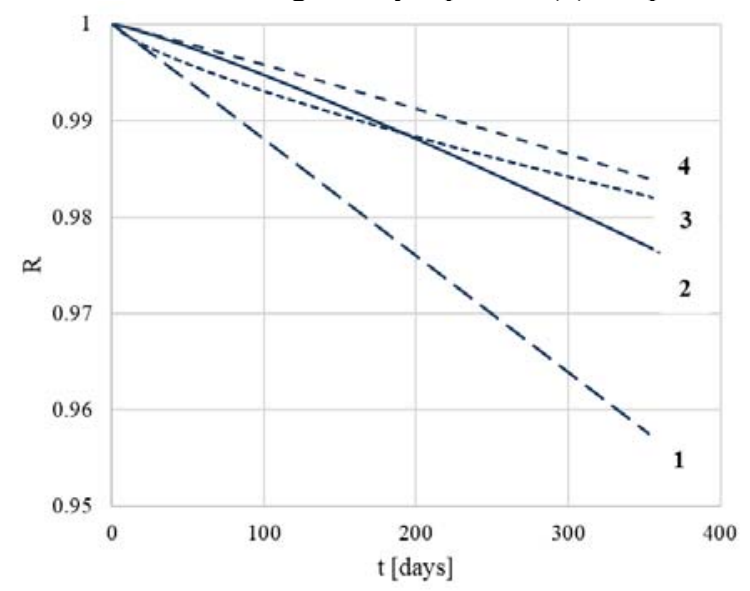

Fig. 3. Wheel failure modes estimated probabilistic models: 1 - wheel flat; 2 - loosened tyre; 3 - scaled wheel; 4 - tyre wear. 
As expected, during the observation period, the decrease in time of the reliability function is as faster as the failure mode absolute frequency occurrence is higher. On the other hand, in the case of a lower value of the shape parameter (see $3^{\text {rd }}$ failure mode) although initially can be observed a significant drop of reliability, a more favourable evolution is obtained subsequently. The higher value of the scale parameter of the $3^{\text {rd }}$ failure mode model also contributes to this evolution.

\section{Conclusion}

Railway wheel reliability is vital from the point of view of traffic safety, thus identifying and analysing the wheel failure modes is essential. Given this, the aim of the present paper was to analyse the wheel failure modes, based on real field data - obtained during a period of a year of operation of freight wagons.

There were recorded 14 wheel failure modes, including a total of 137 failure cases. It was found that the Pareto principle applies to a good extent in the case of the analysed data: the first 4 (out of 14) most frequent failure modes were causing $81 \%$ of the total. Considering the first three failure modes, and grouping into a unique failure mode (the forth) all failures related with tyre wear, there were obtained four major failure modes covering 126 failure cases.

The four main wheel failure modes were analysed using the total time on test transform. The resulting plots have revealed a low curvature, which indicated a low variation in time of the associated failure rates.

There were estimated the probabilistic models for each of the four major wheel failure modes, based on the Weibull distribution. The Weibull probability law was linearized, allowing the use of linear regression to estimate, based on experimental data, the shape and scale parameters. The estimated values of the shape parameter were generally close to 1 , i.e. the failure rates of the four failure modes are close to be constant. It could be concluded, thus, that the behavior of the railway wheel, from the point of view of the analysed failure modes, was close to that of a non-ageing system. An explanation for this was that the data concerning the railway wheel failure were collected in the normal/useful life period. This conclusion is also in line with the general theory regarding the behavior of systems during their normal/useful life.

\section{References}

1. M. Spiroiu, Reliability and maintenance of railway vehicles (in Romanian) (Matrix Rom, Bucharest, 2006)

2. M. A. Spiroiu, Applied Mechanics and Materials, 809-810, 1097-1102 (2015)

3. Y. Liu, L. Liu, B. Stratman, S. Mahadevan, Reliab. Eng. Syst. Safe., 93, 456-467 (2008)

4. Y. Liu, B. Stratman, S. Mahadevan, Int. J. Fatigue, 28, 747-756 (2006)

5. T. A. Zucarelli M. A. Vieira L. A. Moreira Filho, D. A. P. Reis, L. Reis, Proc. Str. Int., 1, 212-217 (2016)

6. Z. X. Liu, H. C. Gu, J. Mater. Eng. Perform., 9, 580-584 (2000)

7. A. Mazzu, L. Solazzi, M. Lancini, C. Petrogalli, A. Ghidini, M. Faccoli, Wear, 342343, 22-32 (2015)

8. J. Lin, J. Pulido, M. Asplund, Reliab. Eng. Syst. Safe., 134, 143-156 (2015)

9. M. A. Spiroiu, MATEC Web Conf., 112, 09008 (2017) 